\title{
Differential regulation of muscle protein turnover in response to emphysema and acute pulmonary inflammation
}

Judith J. M. Ceelen ${ }^{1}$, Annemie M. W. J. Schols ${ }^{1}$, Stefan J. van Hoof ${ }^{2}$, Chiel C. de Theije ${ }^{1}$, Frank Verhaegen² and Ramon C. J. Langen ${ }^{1 *}$

\begin{abstract}
Background: Exacerbations in COPD are often accompanied by pulmonary and systemic inflammation, and associated with increased susceptibility to and prevalence of weight loss and muscle wasting. Muscle mass loss during disease exacerbations may contribute to emphysema-associated muscle atrophy. However, whether pulmonary inflammation in presence of emphysema differentially affects skeletal muscle, including protein synthesis and degradation signaling pathways has not previously been addressed. The aims of this study were to 1) develop a mouse model of disease exacerbation-associated muscle wasting, 2) evaluate whether emphysema and muscle wasting can be monitored non-invasively and 3) assess alterations in muscle protein turnover regulation.
\end{abstract}

Methods: Emphysema was induced by three, weekly intra-tracheal (IT) elastase (E) or vehicle control (vc) instillations, followed by one single IT-LPS bolus (L) or vc instillation to mimic pulmonary inflammation-driven disease exacerbation. Consequently, four experimental groups were defined: vc/vc ('C'), E/vc ('E'), vc/LPS ('L'), E/LPS ('E $+L^{\prime}$ ). Using micro cone-beam CT-scans, emphysema development and muscle mass changes were monitored, and correlated to muscle weight $48 \mathrm{~h}$ after LPS instillation. Protein turnover signaling was assessed in muscle tissue collected $24 \mathrm{~h}$ post LPS instillation.

Results: Micro-CT imaging correlated strongly with established invasive measurements of emphysema and muscle atrophy. Pulmonary inflammation following LPS instillation developed irrespective of emphysema and body and muscle weight were similarly reduced in the ' $L$ ' and ' $E+L$ ' groups. Accordingly, mRNA and protein expression levels of genes of the ubiquitin-proteasome pathway (UPS) and the autophagy-lysosomal pathway (ALP) were upregulated in skeletal muscle following IT-LPS ('L' and ' $E+L$ '). In contrast, mTOR signaling, which controls ALP and protein synthesis, was reduced by pulmonary inflammation (' $L$ ' and ' $E+L$ ') as well as emphysema as a single insult ('E') compared to control.

Conclusion: Changes in lung tissue density and muscle mass can be monitored non-invasively to evaluate emphysema and muscle atrophy longitudinally. Acute loss of muscle mass evoked by pulmonary inflammation is similar in control and emphysematous mice. Although muscle atrophy cues in response to pulmonary inflammation are not altered by emphysema, emphysema itself affects protein synthesis and ALP signaling, which may interfere with muscle mass recovery and impair maintenance of muscle mass in emphysema.

Keywords: Inflammation-induced atrophy, ALP, UPS, Protein synthesis signaling

\footnotetext{
* Correspondence: r.langen@maastrichtuniversity.nl

${ }^{1}$ Department of Respiratory Medicine, Maastricht University Medical Center,

PO box 5800, 6202 AZ Maastricht, The Netherlands

Full list of author information is available at the end of the article
} 


\section{Background}

Chronic obstructive pulmonary disease (COPD) is characterized by persistent airflow obstruction, resulting from inflammation and remodeling of the airways and, in some patients may include development of emphysema [1]. A major extra-pulmonary hallmark of COPD is muscle wasting which has been shown to be an independent predictor of exercise performance [2] and of mortality [3]. Moreover, COPD patients with an emphysematous phenotype appear to be more prone to skeletal muscle wasting [4].

Skeletal muscle mass is determined by cellular processes that include proteolysis and synthesis, but their contribution to muscle wasting in emphysematous COPD patients is still unclear [5]. Acute exacerbations in COPD are a temporary worsening of the disease which accelerate disease progression and decrease survival [6]. COPD patients that suffer from frequent exacerbations are more prone to weight loss and muscle wasting $[7,8]$. Furthermore, Alahmari et al. showed that frequent exacerbations are associated with increased muscle weakness and a decline in exercise capacity [9].

Exacerbations can be triggered by bacterial respiratory infections [10] and are often accompanied by pulmonary and systemic inflammation [11, 12]. Previous studies have shown that pulmonary inflammation evoked by the bacterial component LPS, is sufficient to induce acute loss of skeletal muscle mass $[13,14]$. Furthermore, several studies have shown that inflammation causes a disturbance in muscle protein turnover $[15,16]$.

However, the effects of underlying emphysema on muscle protein turnover regulation and acute pulmonary inflammation-induced muscle atrophy have not been studied. Elastase-induced emphysema is an interesting experimental model as pulmonary inflammation is absent [17], and no changes in muscle mass have been observed [18]. Conversely, smoke-induced emphysema in mice is accompanied by low-grade inflammation and a slight decrease in muscle mass [19], whereas emphysema and a strong pulmonary and systemic inflammatory response in SP-C/TNF transgenic mice is accompanied by a marked reduction in body and muscle weight. Moreover, impaired muscle regeneration observed in these mice suggests that emphysema and inflammation may impair the recovery of atrophied muscle [20].

We postulate that the cumulative effects of acute loss of muscle mass during disease exacerbations and impaired muscle regrowth during stable disease underlies COPD-associated muscle atrophy.

To discern between the effects of inflammation and emphysema during exacerbation we adopted the model of Kobayashi et al., in which a single intratracheal instillation of LPS evokes exacerbation-like features in mice with elastase-induced emphysema [17].
Our first objective was to evaluate muscle wasting in this model of COPD exacerbation, and secondly to assess emphysema and muscle mass changes noninvasively. Emphysematous changes were assessed by quantifying the percentages of low attenuation area (LAA\%) using micro computed tomography $(\mu \mathrm{CT})$, and validated by comparing to the mean linear intercept $(\mathrm{Lm})$, the current golden standard for measuring emphysema. Muscle volumes were also determined using $\mu \mathrm{CT}$ scan analysis, and validated using the skeletal muscle wet weights. The third objective was to investigate protein turnover regulation, including proteolysis and protein synthesis signaling, associated with muscle mass changes.

\section{Methods}

Animals and experimental protocol

This study was approved by the Institutional Animal Care Committee of Maastricht University. Twelve-weekold C57BL/6 J male mice (Charles River Laboratories International, Wilmington, MA) were randomly divided into four groups, each subjected to one of the following pre-treatment/treatment regimens: controls receiving only vehicle control (vc) solutions; vc/vc, designated ' $\mathrm{C}$ '), elastase pre-treated followed by vc treatment (E/vc, designated ' $\mathrm{E}$ '), vc pre-treated and LPS-treated ( $\mathrm{vc} / \mathrm{L}$, designated ' $L$ ') and both elastase pre-treated followed by LPS-treatment $(E / L$, designated ' $E+L$ '). Animals received 3 weekly intratracheal (IT) instillations with $4 \mathrm{U}$ of porcine pancreatic elastase (PPE) (Wako Instruchemie BV, Delfzijl, Netherlands) dissolved in $50 \mu \mathrm{l}$ sterile PBS, or $50 \mu \mathrm{l}$ of PBS alone (vc). 21 days after the last elastase instillation, the degree of emphysema was determined by $\mu \mathrm{CT}$-scan analysis. Then mice received an IT instillation of $2.5 \mu \mathrm{g}$ LPS per gram mouse (Escherichia coli, serotype O55:B5; Sigma, St. Louis, MO) dissolved in $50 \mu \mathrm{l}$ sterile $0.9 \% \mathrm{NaCl}$ or $50 \mu \mathrm{l}$ sterile $0.9 \% \mathrm{NaCl}$ alone (vc). Mice were sacrificed at $24 \mathrm{~h}$ after LPS to assess signaling $(n=$ $8-12$ /group) or $48 \mathrm{~h}$ after LPS to assess muscle atrophy ( $n=11$ /group) [14]. The lungs were rinsed with cell culture medium (i.e., Dulbecco's Modified Eagle's Medium containing $0.5 \%$ FBS, $50 \mathrm{U} / \mathrm{ml}$ penicillin, and $50 \mathrm{mg} / \mathrm{ml}$ streptomycin) to obtain bronchoalveolar lavage fluid (BALf). Alternatively, lungs were isolated and fixed for histological purposes. The soleus, gastrocnemius, tibialis anterior, plantaris, and EDL muscles were collected from both hind limbs, using standardized dissection methods, weighed and stored at $-80{ }^{\circ} \mathrm{C}$ for RNA and protein extraction.

\section{$\mu \mathrm{CT}$ imaging and analysis for non-invasive assessment of emphysema and muscle mass}

Mice were anesthetized with a mixture of air and 4\% isoflurane and scanned using a cone beam, $\mu \mathrm{CT}$ scanner 
(XRAD-225Cx, Precision X-Ray, North Branford, USA) at a dose of 0.28 Gy. $\mu \mathrm{CT}$ images were acquired at 80 $\mathrm{kVp}$ and reconstructed to a 3D image volume with an isotropic voxel spacing of $0.2 \mathrm{~mm}$. Resulting image data were analyzed using SmART-Plan (version 1.3.6) [21]. A selection of the lungs was made and the density of each voxel was plotted in a density histogram. The LAA threshold was set at -426 Hounsfield units (HU). The LAA\% was calculated as the ratio of LAA to the total lung area. To validate $\mu \mathrm{CT}$ evaluation of emphysema, LAA\% values were subsequently correlated to histological assessment of airspace enlargement (described below).

The muscle of the hindlimbs was delineated using SmART-Plan. Muscle volume was determined as the total selected volume minus air and bone (thresholds -460 and $520 \mathrm{HU}$ respectively). Muscle volumes were then converted by using CT to mass density calibration function, established using the same imaging protocol. The HU to density calibration was performed using a small phantom with a diameter of $29.5 \mathrm{~mm}$, with known $3.6 \mathrm{~mm}$ diameter tissue substitute inserts. The tissue substitute inserts were derived from a clinical radiotherapy phantom (Model 467; Gammex RMI, Middleton, WI). A cone-beam CT scan was acquired of this phantom and loaded into SmART-Plan in which all inserts were delineated. The regions of interest for each insert had a volume of $25 \mathrm{~mm}^{3}$. That volume of $25 \mathrm{~mm}^{3}$ is smaller than the volume of the inserts, because a margin was used at the edges of the inserts for structure delineation. Average HU values were determined from the regions of interest. Two linear functions were fitted to the data points, the first conversion function covers all $\mathrm{HU}$ values up to and including the intercept of the two linear functions, and the second function covers all higher $\mathrm{HU}$ values. The parameters of the first linear function fit, for $\mathrm{HU}$ values up to 162 , of the form, density $=\mathrm{A} * \mathrm{HU}+\mathrm{B}$ were: $\mathrm{A}=9.508 * 10^{-4}$ and $\mathrm{B}=0.8405$. The parameters for the second function fit, for HU values of 163 and higher, were: $\mathrm{HU}, \mathrm{A}=2.7191^{*} 10^{-4}$ and $\mathrm{B}=0.9506$. Density of each voxel was calculated, multiplied with the volume of the voxel and summed, generating the mass of the selected muscle. To validate $\mu \mathrm{CT}$ assessment of muscle mass, these values were subsequently correlated to muscle wet weights.

\section{Histological assessment of emphysema}

The lungs were fixated by infusion of $4 \%$ paraformaldehyde through a tracheal cannula according to ATS/ERS guidelines for quantitative assessment of lung structure $[22,23]$. After excision, the lung was immersed in fresh fixative for $24 \mathrm{~h}$. The lung lobes were embedded in paraffin, cut into $4 \mathrm{~mm}$ transverse sections and stained with haematoxylin and eosin staining for histological analysis.
Enlargement of alveolar spaces was determined by quantifying the mean linear intercept (Lm) using image analysis software (Image J 1.33). Sections were scanned using a dotslide light microscopy slide scanner at 100x magnification (Olympus, Hamburg, Germany) and analyzed entirely. Pictures were converted to black (tissue) and white (background) and cutting artifacts or hilar structures (airway or blood vessel with a diameter larger than $50 \mu \mathrm{m})$ were excluded from the analysis. Furthermore, background noise was removed using a threshold of 3 pixels. The Lm was measured over the whole lung section by placing a grid on each pixel line. The mean of all white pixel lines gives the average distance between alveolar surfaces, or the Lm.

\section{Assessment of lung inflammation}

BALfluid was centrifugated $\left(5 \mathrm{~min}\right.$ at $1500 \mathrm{rpm}, 4{ }^{\circ} \mathrm{C}$ ), pelleted cells were resuspended in differentiation medium (i.e., Dulbecco's Modified Eagle's Medium containing $0.5 \%$ FBS, $50 \mathrm{U} / \mathrm{ml}$ penicillin, and $50 \mathrm{mg} / \mathrm{ml}$ streptomycin) and incubated for $3 \mathrm{~h}$ at $37{ }^{\circ} \mathrm{C}$ to obtain conditioned medium $(\mathrm{CM})$. $\mathrm{CM}$ was centrifugated and the supernatant was stored at $-80{ }^{\circ} \mathrm{C}$ until further use. NF- $\mathrm{KB}$ luc-reporter cells were grown on Matrigel (Becton Dickinson Labware, Bedford, MA) and differentiated into myotubes (as described previously) [24]. Myotubes were incubated with $\mathrm{CM}$ for $6 \mathrm{~h}$ at $37^{\circ} \mathrm{C}$, whereafter they were harvested in Reporter lysis buffer (Promega, Madison, USA). Luminescence was determined using a luminometer (Berthold Lumat LB 9507, Belgium).

\section{RNA isolation}

RNA was isolated from homogenized gastrocnemius muscle from mice sacrificed $24 \mathrm{~h}$ post LPS using the RNeasy plus mini kit (Qiagen). cDNA was made with the Tetro cDNA Synthesis kit (GC biotech). Primer sequences of transcripts of interest are provided in Additional file 1: Table S1. The relative DNA starting quantities of the samples were derived using LinRegPCR software (Version 2014.0, Ruijter). The expression of genes of interest was normalized to the geometric average of four reference genes (cyclophilin A, beta-2microglobulin, 18S, RPL13a) by the GeNorm software.

\section{Western blotting}

Gastrocnemius muscle from mice sacrificed $24 \mathrm{~h}$ post LPS was processed and analyzed as previously described [25]. In short, muscle was ground to powder using an $\mathrm{N}_{2}$-cooled steel mortar. The powder ( 20 mg) was lysed in IP-lysis buffer containing protease inhibitors (Complete; Roche Nederland, Woerden, Netherlands), using a rotating blade tissue homogenizer (Polytron homogenizer, Kinematica). Total protein concentration 
of the supernatant was determined with a BCA protein assay kit (Pierce Biotechnology, \#23225, Rockford, IL) according to manufacturer's instructions. Laemmli buffer was added and samples were denatured by heating at $100{ }^{\circ} \mathrm{C}$ for $5 \mathrm{~min}$. Samples were analyzed by western blot. Briefly, $10 \mu \mathrm{g}$ of protein per lane were separated on a CriterionTM XT Precast $4-12 \%$ or $12 \%$ Bis-Tris gel (Bio-Rad Laboratories, Veenendaal, Netherlands) and transferred to a nitrocellulose transfer membrane (BioRad Laboratories) by electroblotting. The membrane was stained with Ponceau $S$ solution $(0.2 \%$ Ponceau $S$ in $1 \%$ acetic acid; Sigma-Aldrich Chemie) to control for protein loading. The membranes were blocked, washed in TBS-Tween ${ }^{0.05 \%}$ and incubated overnight at $4{ }^{\circ} \mathrm{C}$ with primary antibodies. A list of used antibodies is provided in Additional file 1: Table S2. All antibodies were diluted $1 / 1000$ in TBS-Tween ${ }^{0.05}$. Blots were then washed and probed with a horseradish peroxidase-conjugated secondary antibody (Vector Laboratories, Burlingame, CA) and visualized with chemiluminescence (Supersignal West Pico or Femto Chemiluminescent Substrate; Pierce Biotechnology) in a LAS-3000 Luminescent Image analyzer (Fujifilm, Tokyo, Japan). Bands were quantified using the Quantity One software (Bio-Rad, version 4.5.0). All data were corrected for protein loading as determined after Ponceau S staining.

\section{Statistical analysis}

Data are shown as means $\pm \mathrm{SE}$. Comparisons were computed using SPSS version 22.0. For assessment of significance between groups and genotypes an independent samples $T$-test was used. A $p$ value $<0.05$ was considered statistically significant. Asterisks above a bar refer to a comparison with the respective control (L vs. C and E + $\mathrm{L}$ vs. E). An asterisk above a line refers to a comparison between $\mathrm{E}$ vs. $\mathrm{C}$ or $\mathrm{E}+\mathrm{L}$ vs. L. $0.05<p<0.1$ was considered a trend.

\section{Results}

\section{Assessment and validation of emphysema in mice using} $\mu \mathrm{CT}$

Pulmonary emphysema is characterized by the destruction of alveolar walls, leading to enlargement of alveolar spaces [1]. To verify development of emphysema in response to intratracheal elastase, lungs were isolated and lung sections were stained. Alveolar spaces were considerably enlarged in response to elastase (Fig. 1a). In order to determine development of emphysema non-invasively in vivo, $\mu \mathrm{CT}$ scans were made and analyzed, and the percentage of low attenuation area (LAA\%) was calculated for each lung (Fig. 1b). Mice that received elastase had a significantly higher LAA\% than the control mice (Fig. 1c). To verify this method, emphysema was also assessed as airspace enlargement by histological determination of the mean linear intercept (Lm) in a subset of lungs. LAA\% and Lm highly correlated (Fig. 1d).

To determine whether emphysema affected pulmonary inflammation in response to IT-LPS, NF- $\mathrm{KB}$ luc-reporter cells were incubated with medium conditioned by cells obtained from BALfluid. No differences were observed in the basal inflammatory state, and the increase in luciferase activity indicated a similar extent of pulmonary
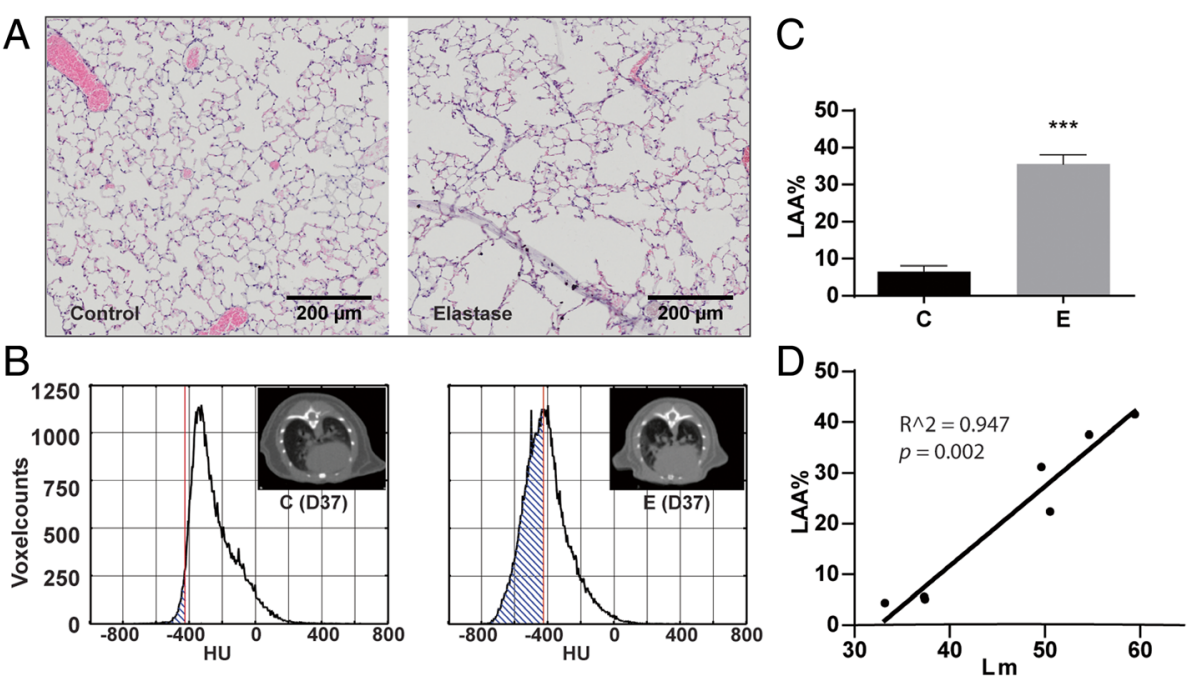

Fig. 1 Development of emphysema after instillation with elastase. Mice were intratracheally instilled with elastase to induce emphysema. a lungs were isolated $48 \mathrm{~h}$ after IT-LPS/NaCl and sections were stained with H\&E. $\mathbf{b} \mu \mathrm{CT}$ scans were analyzed creating a density histogram of the voxels in the selected lungs. The LAA threshold was set to $-426 \mathrm{HU}$. The LAA\% was calculated using the ratio of the total LAA volume (shaded area) to the total lung volume. The upper right corner shows a representative $\mu \mathrm{CT}$ image. c Changes in LAA\% after IT-elastase, as determined by $\mu \mathrm{CT}$ scan analysis. $n=46$ per group. $\mathbf{d}$ Correlation between $\mu \mathrm{CT}$-derived LAA\% and the Lm (both determined $48 \mathrm{~h}$ after NaCl or LPS), $n=7 .{ }^{* * *} p<0.001$ 
inflammation in control and emphysematous mice post IT-LPS (Additional file 2: Figure S1).

\section{Skeletal muscle atrophy in response to pulmonary inflammation in control and emphysematous mice}

After LPS, a rapid decrease in body- (Additional file 3: Figure S2) and skeletal muscle wet weights (Fig. 2a) was observed for control and emphysematous mice. Prior to collection of tissues, $\mu \mathrm{CT}$ scans were made $48 \mathrm{~h}$ after LPS and muscle volume was determined to evaluate whether changes in muscle mass can be measured noninvasively (Fig. 2b). A similar magnitude of decrease of $10-15 \%$ was observed in $\mu \mathrm{CT}$-derived muscle mass (Fig. 2b) compared to muscle wet weights (Fig. 2a). Moreover, $\mu \mathrm{CT}$-derived muscle mass and combined muscle wet weights highly correlated (Fig. 2c).

\section{Alterations in protein turnover signaling in muscle of emphysematous mice with and without pulmonary inflammation}

Activation and involvement of effectors of UPS-mediated proteolysis in pulmonary inflammation-induced muscle atrophy has been demonstrated previously [13, 14]. mRNA transcript levels of the E3 ubiquitin ligases MuRF1, Atrogin-1 and SMART were significantly increased after LPS compared to vc instillation in both control as well as emphysematous mice (Fig. 3a-c), indicating activation of upstream UPS signaling by pulmonary inflammation. The expression of Atrogin-1 and MuRF1 is regulated by activation of FoXO family of transcription factors. Phosphorylation on Thr24 by Akt inactivates FoXO1 resulting in nuclear export and inhibition of transcriptional activity [26]. Although the FoXO1 p/total ratio did not change (Fig. 3e), levels of phosphorylated FoXO1 were increased (Fig. 3f), and accompanied by a similar increase in total FoXO1 protein abundance (Fig. 3g). mRNA transcript levels of FoXO1 were significantly increased (Fig. 3d), suggesting increased de novo FoXO1 protein synthesis.

Besides UPS, there is a role for ALP in muscle proteolysis in the determination of protein turnover signaling.
During active autophagy the cytosolic form of LC3 (LC3B-I) is conjugated to the lipidated form (LC3B-II), resulting in recruitment to the autophagosomal membrane [27]. In both control and emphysematous mouse muscle, the LC3B-II/I ratio was significantly increased (Fig. 4a), which is explained by the decreasing levels of LC3B-I (Fig. 4c) and increasing levels of LC3B-II (Fig. 4b). This was accompanied by increased mRNA levels of LC3B after LPS (Fig. 4d). Interestingly, both LC3B-I as well as LC3B-II levels were significantly higher in emphysematous mice compared to control, although this was not reflected in the ratio. ULK1 ser757 phosphorylation by mTOR prevents autophagosome formation [28]. In both control and emphysematous mouse muscle, the ULK1 ratio was significantly decreased (Fig. 4e), due to decreased levels of phosphorylated ULK1 (Fig. 4f). Total levels of ULK1 were increased after LPS (Fig. 4g), suggesting stabilization of the protein. p62 can bind ubiquitinated proteins and LC3B, thereby targeting the autophagosome and facilitating clearance of ubiquitinated proteins [29]. There was no change in p62 protein levels, however mRNA levels increased significantly after LPS (Fig. 4i-j), suggesting that autophagy-mediated turnover of p62 is increased after LPS. Also mRNA levels of Gabarapl, a ubiquitin-like protein required for the formation of autophagosomal membranes, and BNIP, a membrane-bound receptor that can bind LC3B or Gabarapl, were increased after LPS (Fig. 4k-l).

Indices of protein synthesis signaling, including the ratio of p70S6, S6 and 4EBP1 decreased significantly after LPS (Fig. 5a, e, i and k), which is explained by a decrease in phosphorylation (Fig. 5b, f, j, l). Interestingly, phosphorylation of S6 and p70S6 (trend only) were already decreased in emphysematous mice compared to control, which is also reflected in the ratio. This suggests that in emphysematous mice, there is already a decrease in protein synthesis signaling independent from pulmonary inflammation. mTOR is phosphorylated at ser2448 via the PI3K/Akt pathway and plays a key role in muscle
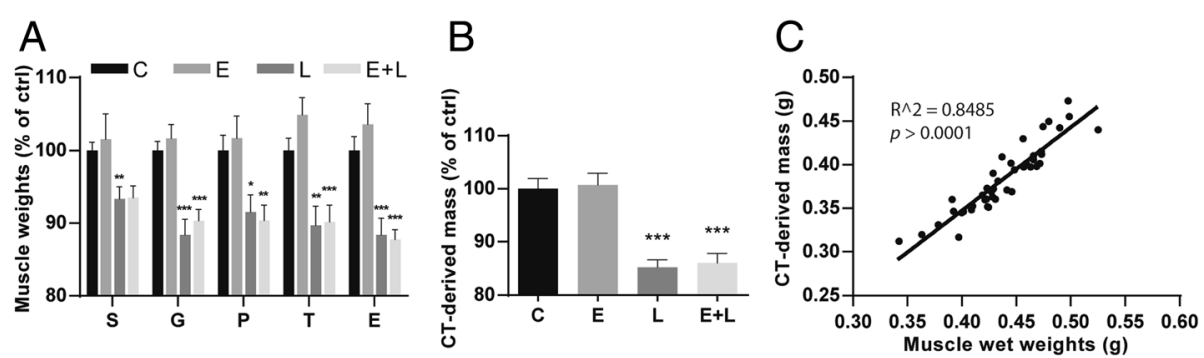

Fig. 2 Pulmonary inflammation causes muscle atrophy in control and emphysematous mice. Mice were intratracheally instilled with elastase to induce emphysema or vc, followed by a single bolus of LPS or vc. a $48 \mathrm{~h}$ after LPS, mice were sacrificed and skeletal muscle wet weights were measured and corrected for tibia length. $\mathbf{b} 48 \mathrm{~h}$ after LPS or vc, $\mu \mathrm{CT}$ scans were made and muscle mass was determined. c Correlation between $\mu \mathrm{CT}$-derived muscle mass and combined muscle wet weights. ${ }^{*} p<0.05,{ }^{* *} p<0.01$, ${ }^{* *} p<0.001 . n=11-23$ per group 

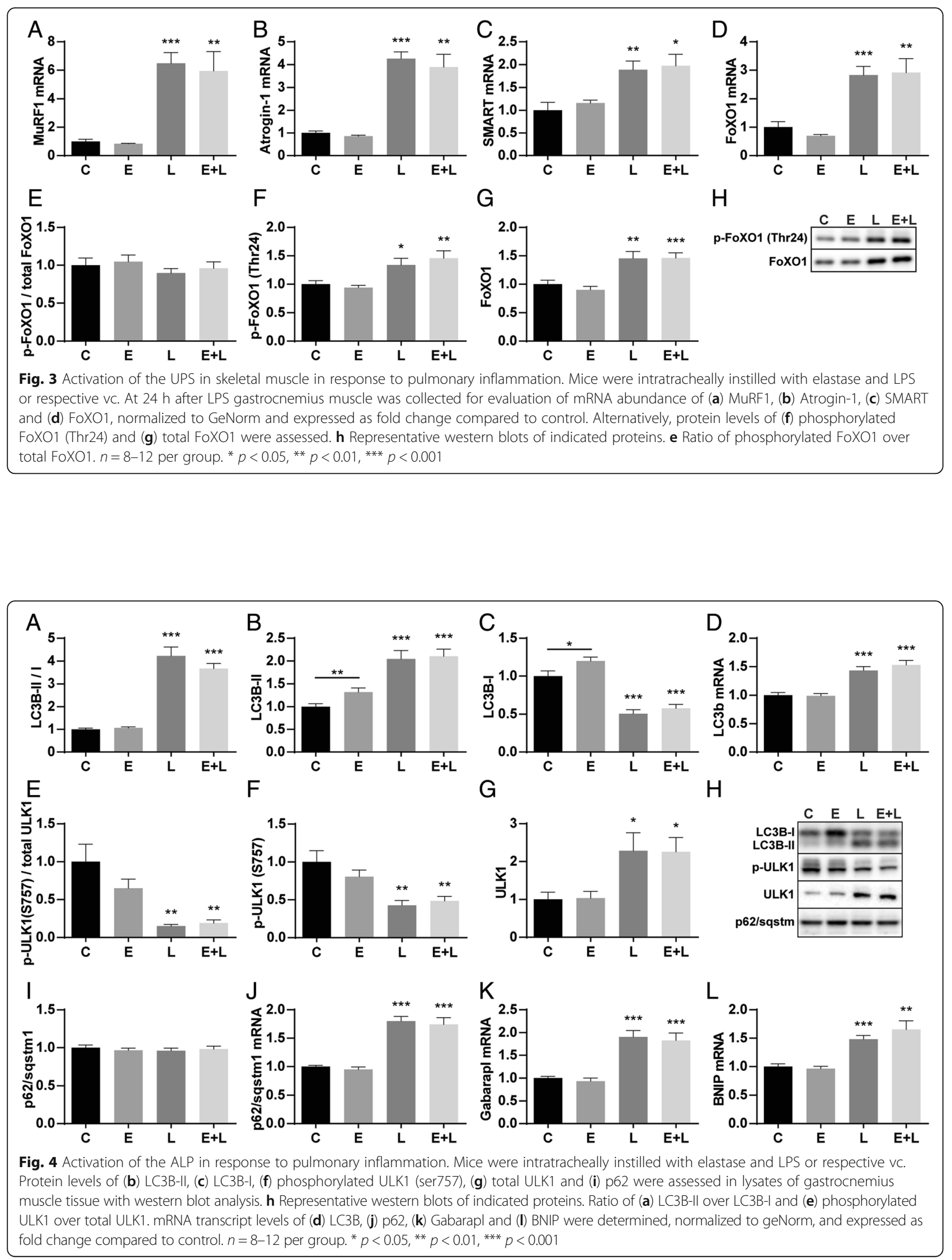


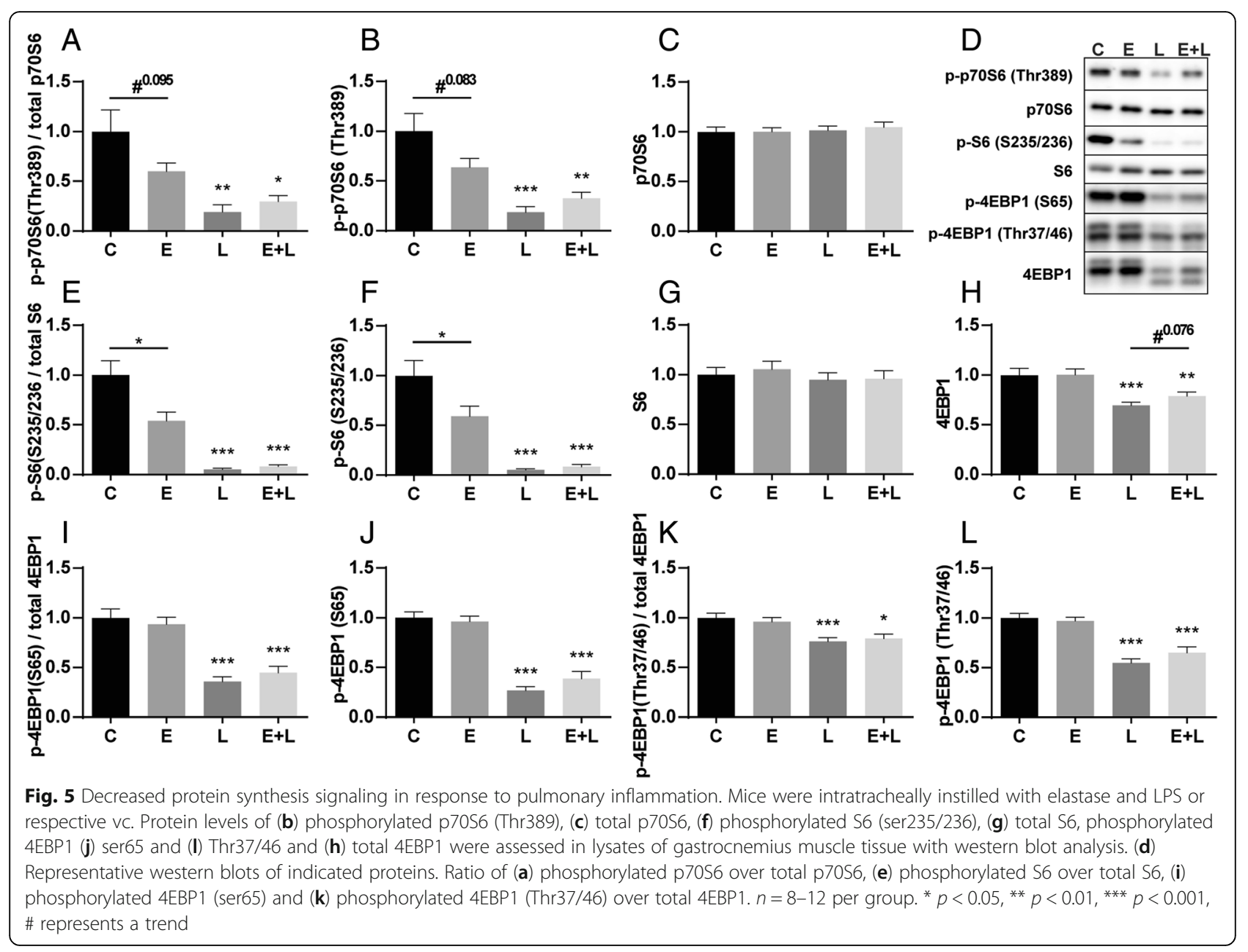

protein turnover. Apart from a slight trend towards decreased phosphorylation of mTOR after LPS, no changes were observed (Fig. 6a-c). The Akt ratio was decreased in the control mice after LPS (Fig. 6e), which is explained by decreased Akt S473 phosphorylation (Fig. 6f). mTORC1 activity can be regulated by REDD1, which stimulates the inhibitory actions of TSC2 on mTORC1 [30]. mRNA transcript levels of REDD1 were highly increased acutely after LPS instillation (Fig. $6 \mathrm{~h}$ ), in line with the downregulation of targets downstream of mTOR after LPS.

\section{Discussion}

\section{Non-invasive detection of emphysema and muscle atrophy}

The course of muscle wasting in the emphysematous phenotype of COPD remains unresolved. As demonstrated in this work, the use of $\mu \mathrm{CT}$ scan analysis allows non-invasive monitoring of the development of emphysema and assessment of skeletal muscle mass. Several groups have used non-invasive measurements to determine emphysema [17, 31, 32], and the significant correlation between the LAA\% measured by $\mu \mathrm{CT}$ and the Lm shown here further confirms the reliability of $\mu \mathrm{CT}$ as a tool for measuring emphysema. Importantly, in addition to providing an accurate measure for emphysema in vivo, $\mu \mathrm{CT}$ scan analysis showed to be reliable in determining muscle mass non-invasively, considering the high and significant correlation between $\mu \mathrm{CT}$ derived muscle mass and skeletal muscle wet weights. This will allow longitudinal assessment of muscle atrophy to address the impact of disease exacerbations.

\section{Skeletal muscle atrophy in response to pulmonary inflammation in the presence of emphysema}

Bacterial infections may account for $50 \%$ of all exacerbations in COPD [10]. Using LPS to induce an acute pulmonary inflammation in mice with elastase-induced emphysema, emphysema progression and immune cell infiltration have been postulated, similar to COPD patients with exacerbations [17]. Although previous studies have shown that acute lung inflammation is sufficient to induce loss of skeletal muscle mass [13, 14], these concern LPS instillations in healthy mice. Since protease 


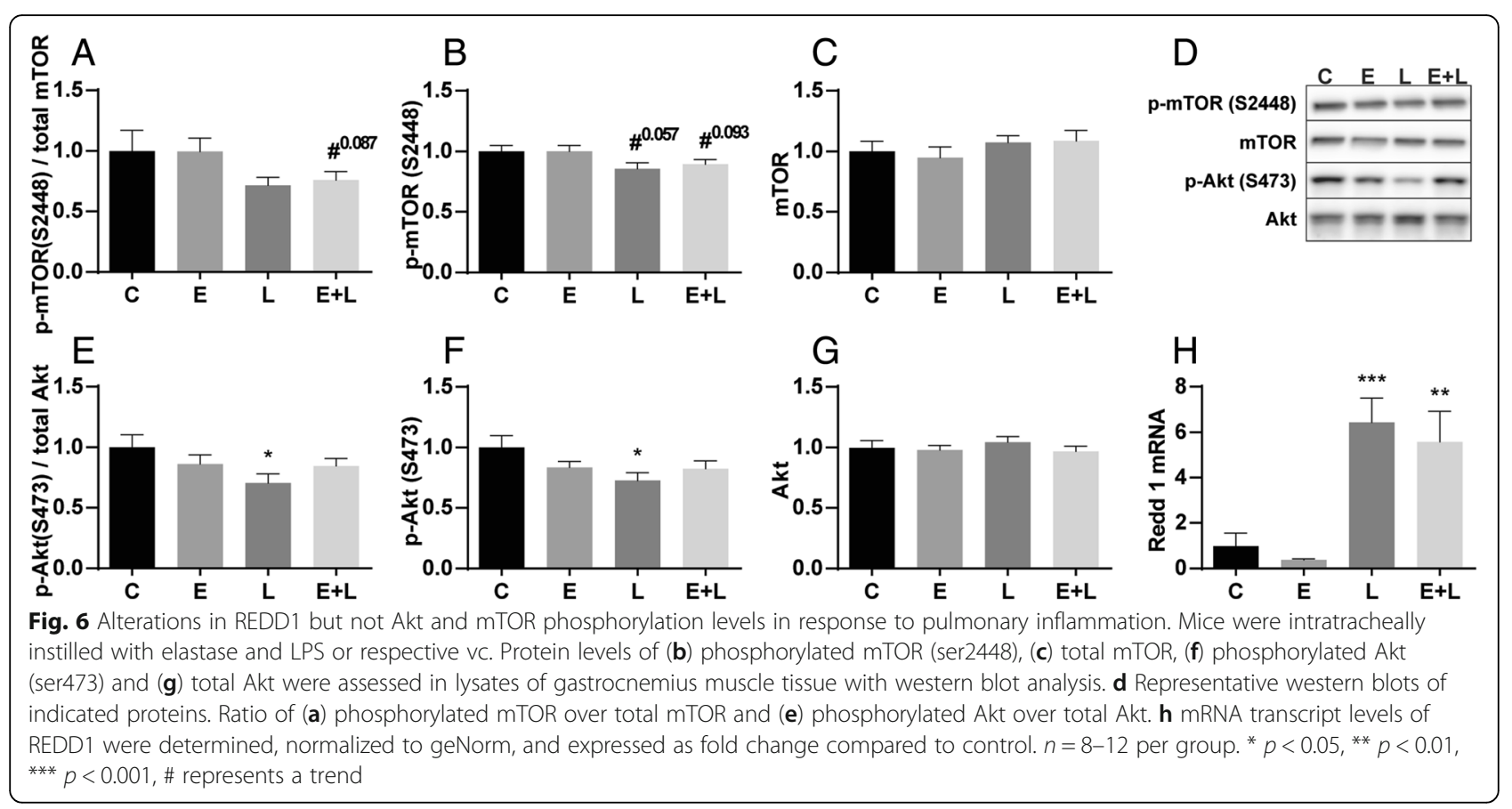

imbalance-induced COPD affects the local immune response [33], pulmonary inflammation may develop differently in the emphysematous lung. However, our data suggests that the pulmonary inflammatory response to LPS is similar in naïve and emphysematous mice. Since emphysema may aggravate the systemic consequences of pulmonary inflammation via e.g., deterioration of gas exchange and subsequent hypoxemia, a synergistic or additive effect of muscle hypoxia [25] and inflammatory signaling on muscle atrophy could be anticipated. However, emphysematous mice loose the same amount of body- and muscle weight as healthy mice after acute pulmonary inflammation. This implies that the mechanistic insights previously acquired with the IT-LPS model in non-emphysematous mice $[13,14]$ very likely apply to the compound model in its current form as well, although systemic effects of interactions between emphysema and pulmonary inflammation may become apparent when a more moderate inflammatory response is evoked. Conversely, in line with previous research [18], there was no evidence of muscle wasting following elastase-induced emphysema. This contrasts the decreases in body and muscle weights described in models of smoke-induced or chronic inflammation-induced emphysema [19, 20], which may suggest that some degree of pulmonary inflammation is required to induce muscle wasting. Correspondingly, no evidence of lung inflammatory signaling or muscle wasting could be detected in emphysematous mice at baseline. The compound model of disease exacerbation characterized in our study will be useful to discern the effects of and interactions between emphysema and pulmonary inflammation on skeletal muscle wasting and other systemic consequences of COPD.

\section{Muscle protein turnover signaling in response to pulmonary inflammation}

The observed muscle wasting likely involves a contribution of increased proteolysis and decreased protein synthesis signaling, with subtle differences in skeletal muscle of healthy compared to emphysematous mice. Increases in expression of E3 ubiquitin ligases MuRF1, Atrogin-1 and SMART indicate that UPS-mediated proteolysis is activated after pulmonary inflammation. Some evidence of muscle ALP activation in pulmonary inflammation was provided in previous work [14, 34], where the expression of autophagy-related genes including LC3B, Bnip3 and GabarapL1 was significantly upregulated after LPS. Here we demonstrate an increased LC3B-II/I ratio, decreased phosphorylation of ULK1 and increased GabarapL, Bnip and p62 mRNA levels, demonstrating similar activation of ALP in skeletal muscle of emphysematous and control mice after pulmonary inflammation.

In emphysematous mice, phosphorylation of p70S6 and S6, as well as p-4EBP1 decreases acutely after LPS similar to control mice with acute pulmonary inflammation. This downregulation of protein synthesis signaling coincides with the downregulation of ULK1 Ser757 phosphorylation, implying that coordination of autophagy initiation and inhibition of protein synthesis during pulmonary inflammation occurs at the level of reduced mTOR activity. 


\section{Alterations in muscle protein turnover signaling in presence of emphysema}

Increased muscle proteolysis as the basis of muscle wasting in COPD has been suggested in a number of reports. However, this interpretation was either based on indirect measurements [35] or signaling [36] of proteolysis, or did not differentiate between the emphysematous and other phenotypes. Conversely, reduced muscle and whole body protein synthesis was suggested by direct measurements in emphysema patients [37]. In line with this, our current findings reveal impaired protein synthesis signaling based on the decreased phosphorylation levels of p70S6 and S6 in skeletal muscle of emphysematous mice at baseline. Interestingly, phosphorylation of ULK1 was also slightly decreased in emphysematous mice, suggesting reduced mTOR activity independent of Akt-mTOR signaling as levels of phosphorylated Akt (S473) or mTOR (S2448) did not show any differences. Moreover, significantly increased levels of LC3B-I and -II, accompanied by unaltered LC3B mRNA levels, are indicative of decreased LC3B-I to -II conversion and decreased breakdown of LC3B-II, and alterations in autophagy. These data suggest that protein synthesis signaling as well as ALP regulation is already affected in emphysematous mice.

Differences in protein synthesis signaling may contribute to depressed muscle protein synthesis at baseline [37] or in response to anabolic triggers including exercise as was previously demonstrated [38] in patients with emphysema. Although this does not affect muscle mass in emphysematous mice in the timeframe assessed here, this may ultimately culminate in muscle atrophy when sustained for longer periods or as a consequence of an impaired capacity to recover from loss of muscle mass [39].

\section{Conclusion}

In conclusion, micro-CT scan analysis is a reliable method for non-invasive assessment of emphysema and changes in muscle mass, allowing longitudinal monitoring in mice. Skeletal muscle atrophy and changes in muscle protein turnover signaling as systemic consequences of pulmonary inflammation are not altered in emphysematous mice.

\section{Additional files}

Additional file 1: Table S1. Sequences of primers used for RT-qPCR to assess expression of the indicated genes. Table S2. Antibodies used for western blot. (DOCX 17 kb)

Additional file 2: Figure S1. Similar pulmonary inflammation in control and emphysematous mice following IT-LPS instillation. Mice were intra-tracheally instilled with elastase to induce emphysema or VC, followed by a single bolus of LPS or vc. Lungs were lavaged to obtain BALfluid ( $n=11$ or 12/group), and cells isolated from the BALf were used to produce conditioned medium. NF-kB luciferase activity was measured in lysates prepared from $\mathrm{C} 2 \mathrm{C} 12$ myotubes after stimulation with conditioned medium. (TIF $774 \mathrm{~kb}$ )
Additional file 3: Figure S2. Similar loss of bodyweight in control and emphysematous mice following IT-LPS instillation. Mice were intra-tracheally instilled with elastase to induce emphysema or Vc, followed by a single bolus of LPS or vc. Changes in body weight (0 h $n=21-23,24 \mathrm{~h} n=21-23,48 \mathrm{~h} n=10$ or 11) after IT-LPS were measured and expressed as a percentage of their respective IT-NaCl time control. (TIF $1284 \mathrm{~kb}$ )

\section{Abbreviations}

MCT: Micro computed tomography; ALP: Autophagy-lysosomal pathway; BALf: Bronchoalveolar lavage fluid; C: Control; CM: Conditioned medium; COPD: Chronic obstructive pulmonary disease; E: Elastase-treated; E + L: Elastase- and LPS-treated; HU: Hounsfield units; IT: Intra-tracheal; L: LPS-treated; LAA\%: Percentage of low attenuation area; Lm: Mean linear intercept; PPE: Porcine pancreatic elastase; UPS: Ubiquitin-proteasome pathway; vc: Vehicle control

\section{Acknowledgements}

The macros for Lm analysis with ImageJ were kindly provided by Pieter Leermakers.

\section{Funding}

This study was supported by a grant from the Lung Foundation Netherlands (3.2.11.036).

\section{Availability of data and materials \\ Please contact author for data requests.}

\section{Authors' contributions}

JC was responsible for the experimental design, execution of the experimental protocol, carrying out analysis and data interpretation, and conception of the manuscript. AS was involved in the conception of the study and the manuscript. SH created the CT-analysis program and wrote the Matlab scripts. CT was responsible for the muscle dissections and tissue processing. FV was responsible for the CT-scanner and involved in the conception of the study. RL was responsible for conception of the study and the manuscript. All authors have read and approved the final manuscript.

Competing interests

The authors declare that they have no competing interests.

Consent for publication

Not applicable

Ethics approval

This study was approved by the Institutional Animal Care Committee of Maastricht University.

\section{Publisher's Note}

Springer Nature remains neutral with regard to jurisdictional claims in published maps and institutional affiliations.

\section{Author details}

${ }^{1}$ Department of Respiratory Medicine, Maastricht University Medical Center, PO box 5800, 6202 AZ Maastricht, The Netherlands. ${ }^{2}$ Department of Radiation Oncology (MaastRO), Maastricht University Medical Center, Maastricht, The Netherlands.

Received: 13 September 2016 Accepted: 3 March 2017

Published online: 02 May 2017

\section{References}

1. Rabe KF, Hurd S, Anzueto A, Barnes PJ, Buist SA, Calverley P, Fukuchi Y, Jenkins C, Rodriguez-Roisin R, van Weel C, et al. Global strategy for the diagnosis, management, and prevention of chronic obstructive pulmonary disease: GOLD executive summary. Am J Respir Crit Care Med. 2007;176:532-55.

2. Engelen MP, Schols AM, Does JD, Wouters EF. Skeletal muscle weakness is associated with wasting of extremity fat-free mass but not with airflow obstruction in patients with chronic obstructive pulmonary disease. Am J Clin Nutr. 2000;71:733-8. 
3. Schols AMWJ, Slangen J, Volovics L, Wouters EFM. Weight loss is a reversible factor in the prognosis of chronic obstructive pulmonary disease. Am J Respir Crit Care Med. 1998;157:1791-7.

4. Vanfleteren LEGW, Spruit MA, Groenen M, Gaffron S, van Empel VPM, Bruijnzeel PLB, Rutten EPA, Op't Roodt J, Wouters EFM, Franssen FME. Clusters of comorbidities based on validated objective measurements and systemic inflammation in patients with chronic obstructive pulmonary disease. Am J Respir Crit Care Med. 2013;187:728-35.

5. Langen RC, Gosker HR, Remels AH, Schols AM. Triggers and mechanisms of skeletal muscle wasting in chronic obstructive pulmonary disease. Int J Biochem Cell Biol. 2013;45:2245-56.

6. Soler-Cataluna JJ, Martinez-Garcia MA, Roman Sanchez P, Salcedo E, Navarro $\mathrm{M}$, Ochando R. Severe acute exacerbations and mortality in patients with chronic obstructive pulmonary disease. Thorax. 2005;60:925-31.

7. Crul T, Testelmans D, Spruit MA, Troosters T, Gosselink R, Geeraerts I, Decramer M, Gayan-Ramirez G. Gene expression profiling in vastus lateralis muscle during an acute exacerbation of COPD. Cell Physiol Biochem. 2010;25:491-500.

8. Rubinsztajn R, Przybylowski T, Maskey-Warzechowska M, Karwat K, PaplinskaGoryca M, Nejman-Gryz P, Chazan R. Effect of exacerbation frequency on body composition and serum ghrelin and adiponectin concentrations in patients with chronic obstructive pulmonary disease. Pol Arch Med Wewn. 2014;124:403-9.

9. Alahmari AD, Kowlessar BS, Patel AR, Mackay AJ, Allinson JP, Wedzicha JA, Donaldson GC. Physical activity and exercise capacity in patients with moderate COPD exacerbations. Eur Respir J. 2016;48:340-9.

10. Ko FW, Chan KP, Hui DS, Goddard JR, Shaw JG, Reid DW, Yang IA. Acute exacerbation of COPD. Respirology. 2016;21:1152-65.

11. Oudijk EJ, Lammers JW, Koenderman L. Systemic inflammation in chronic obstructive pulmonary disease. Eur Respir J Suppl. 2003;46:5s-13.

12. Perera WR, Hurst JR, Wilkinson TM, Sapsford RJ, Mullerova H, Donaldson GC, Wedzicha JA. Inflammatory changes, recovery and recurrence at COPD exacerbation. Eur Respir J. 2007;29:527-34.

13. Files DC, D'Alessio FR, Johnston LF, Kesari P, Aggarwal NR, Garibaldi BT, Mock JR, Simmers JL, DeGorordo A, Murdoch J, et al. A critical role for muscle ring finger-1 in acute lung injury-associated skeletal muscle wasting. Am J Respir Crit Care Med. 2012;185:825-34.

14. Langen RC, Haegens A, Vernooy JH, Wouters EF, de Winther MP, Carlsen H, Steele C, Shoelson SE, Schols AM. NF-kappaB activation is required for the transition of pulmonary inflammation to muscle atrophy. Am J Respir Cell Mol Biol. 2012;47:288-97.

15. Steiner JL, Crowell KT, Kimball SR, Lang CH. Disruption of REDD1 gene ameliorates sepsis-induced decrease in mTORC1 signaling but has divergent effects on proteolytic signaling in skeletal muscle. Am J Physiol-Endocrinol Metab. 2015;309:E981-94.

16. Vary TC, Kimball SR. Sepsis-induced changes in protein synthesis: differential effects on fast- and slow-twitch muscles. Am J Physiol. 1992;262:C1513-9.

17. Kobayashi S, Fujinawa R, Ota F, Kobayashi S, Angata T, Ueno M, Maeno T, Kitazume S, Yoshida K, Ishii T, et al. A single dose of lipopolysaccharide into mice with emphysema mimics human chronic obstructive pulmonary disease exacerbation as assessed by micro-computed tomography. Am J Respir Cell Mol Biol. 2013;49:971-7.

18. Xiao-lei Z, Bao-sen P, Xiao-li H, Jun W, Chen W. Oxidative stress and peripheral skeletal muscle dysfunction in rats with emphysema. Chin Med J. 2010;123:40-4

19. Gosker HR, Langen RC, Bracke KR, Joos GF, Brusselle GG, Steele C, Ward KA, Wouters EF, Schols AM. Extrapulmonary manifestations of chronic obstructive pulmonary disease in a mouse model of chronic cigarette smoke exposure. Am J Respir Cell Mol Biol. 2009:40:710-6.

20. Langen RC, Schols AM, Kelders MC, van der Velden JL, Wouters EF, JanssenHeininger YM. Muscle wasting and impaired muscle regeneration in a murine model of chronic pulmonary inflammation. Am J Respir Cell Mol Biol. 2006:35:689-96.

21. van Hoof SJ, Granton PV, Verhaegen F. Development and validation of a treatment planning system for small animal radiotherapy: SmART-Plan. Radiother Oncol. 2013;109:361-6.

22. Hsia CCW, Hyde DM, Ochs M, Weibel ER. An official research policy statement of the American thoracic society/European respiratory society: standards for quantitative assessment of lung structure. Am J Respir Crit Care Med. 2010;181:394-418.
23. Ochs M, Mühlfeld C. Quantitative microscopy of the lung: a problem-based approach. Part 1: basic principles of lung stereology. Am J Physiol Lung Cell Mol Physiol. 2013;305:L15-22.

24. Langen RCJ, Schols AMWJ, Kelders MCJM, Wouters EFM, Janssen-Heininger YMW. Enhanced myogenic differentiation by extracellular matrix is regulated at the early stages of myogenesis. In Vitro Cell Dev Biol Anim. 2003;39:163-9.

25. de Theije CC, Langen RC, Lamers WH, Schols AM, Kohler SE. Distinct responses of protein turnover regulatory pathways in hypoxia- and semistarvationinduced muscle atrophy. Am J Physiol Lung Cell Mol Physiol. 2013;305:L82-91.

26. Stitt TN, Drujan D, Clarke BA, Panaro F, Timofeyva Y, Kline WO, Gonzalez M, Yancopoulos GD, Glass DJ. The IGF-1/PI3K/Akt pathway prevents expression of muscle atrophy-induced ubiquitin ligases by inhibiting FOXO transcription factors. Mol Cell. 2004;14:395-403.

27. Tanida I, Ueno T, Kominami E. Human light chain 3/MAP1LC3B is cleaved at its carboxyl-terminal Met121 to expose Gly120 for lipidation and targeting to autophagosomal membranes. J Biol Chem. 2004;279:47704-10.

28. Castets P, Ruegg MA. MTORC1 determines autophagy through ULK1 regulation in skeletal muscle. Autophagy. 2013;9:1435-7.

29. Pankiv $S$, Clausen TH, Lamark T, Brech A, Bruun J-A, Outzen H, Øvervatn A, Bjørkøy G, Johansen T. p62/SQSTM1 binds directly to Atg8/LC3 to facilitate degradation of ubiquitinated protein aggregates by autophagy. J Biol Chem. 2007:282:24131-45.

30. Shimizu N, Yoshikawa N, Ito N, Maruyama T, Suzuki Y, Takeda S, Nakae J, Tagata Y, Nishitani S, Takehana K, et al. Crosstalk between glucocorticoid receptor and nutritional sensor mTOR in skeletal muscle. Cell Metab. 2011;13:170-82.

31. Artaechevarria X, Blanco D, de Biurrun G, Ceresa M, Pérez-Martín D, Bastarrika G, de Torres JP, Zulueta JJ, Montuenga LM, Ortiz-de-Solorzano C, MunozBarrutia A. Evaluation of micro-CT for emphysema assessment in mice: comparison with non-radiological techniques. Eur Radiol. 2011;21:954-62.

32. Ford NL, Martin EL, Lewis JF, Veldhuizen RA, Holdsworth DW, Drangova M. Quantifying lung morphology with respiratory-gated micro-CT in a murine model of emphysema. Phys Med Biol. 2009;54:2121-30.

33. Baraldo S, Turato G, Lunardi F, Bazzan E, Schiavon M, Ferrarotti I, Molena B, Cazzuffi R, Damin M, Balestro E, et al. Immune activation in al-antitrypsindeficiency emphysema. Beyond the protease-antiprotease paradigm. Am J Respir Crit Care Med. 2014;191:402-9.

34. Doyle A, Zhang G, Abdel Fattah EA, Eissa NT, Li YP. Toll-like receptor 4 mediates lipopolysaccharide-induced muscle catabolism via coordinate activation of ubiquitin-proteasome and autophagy-lysosome pathways. FASEB J. 2011;25:99-110.

35. Ubhi BK, Riley JH, Shaw PA, Lomas DA, Tal-Singer R, MacNee W, Griffin JL, Connor SC. Metabolic profiling detects biomarkers of protein degradation in COPD patients. Eur Respir J. 2012;40:345-55.

36. Puig-Vilanova E, Rodriguez DA, Lloreta J, Ausin P, Pascual-Guardia S, Broquetas J, Roca J, Gea J, Barreiro E. Oxidative stress, redox signaling pathways, and autophagy in cachectic muscles of male patients with advanced COPD and lung cancer. Free Radic Biol Med. 2015;79:91-108.

37. Morrison WL, Gibson JN, Scrimgeour C, Rennie MJ. Muscle wasting in emphysema. Clin Sci (Lond). 1988;75:415-20.

38. Engelen MP, Deutz NE, Mostert R, Wouters EF, Schols AM. Response of whole-body protein and urea turnover to exercise differs between patients with chronic obstructive pulmonary disease with and without emphysema. Am J Clin Nutr. 2003;77:868-74.

39. Pansters NA, Schols AM, Verhees KJ, de Theije CC, Snepvangers FJ, Kelders MC, Ubags ND, Haegens A, Langen RC. Muscle-specific GSK-3beta ablation accelerates regeneration of disuse-atrophied skeletal muscle. Biochim Biophys Acta. 1852;2015:490-506. 